\title{
Kooperation mit dem British Institute of Radiology
}

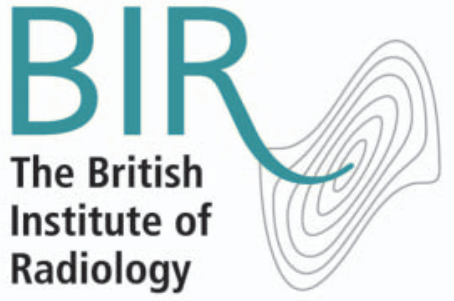

Im April 2018 schlossen sich die Deutsche Röntgengesellschaft (DRG) hat sich mit dem British Institute of Radiology (BIR) und weiteren radiologischen Gesellschaften weltweit zum BIR World Partner Network zusammen. Weltweit soll so das Wissen rund um die radiologische Bildgebung bei Radiologinnen, Medizinisch-Technischen Assistenten und Medizinphysikern gemeinsam gefördert werden.

DRG-Mitglieder erhalten seitdem mit dem monatlichen Newsletter exklusiv Zugang zu Fachinhalten, die nach Veröffentlichung drei Monate online zur Verfügung stehen. Dazu gehören Webinare, beispielsweise zur Diagnose von Lungenerkrankungen, oder auch Fachartikel des British Journal of Radiology, etwa zu Traumata bei älteren Patienten.

Folgende Themen stehen Ihnen als DRGMitglied bislang zur Verfügung:

- Image once, print thrice? Three-dimensional printing of replacement parts (review article)

- Tips on diagnosing fibrosing lung disease (webinar)

- The Role of Emergency Radiologists in the Management of Polytraumatized Patients (webinar)

- Trauma in the elderly patient (article)

- Safety of working patterns among UK neuroradiologists (article)

- CT in Diffuse Interstitial Lung Disease: Survival Guide and Case-Based Discussion (webinar)

- Machine learning for the quantitive analysis of medical imaging/X-ray (webinar)

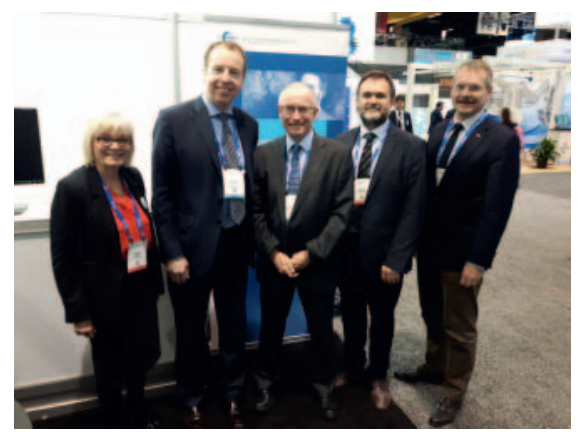

BIR trifft DRG (v.l.n.r.): Carole Cross (BIR, Leiterin Mitgliederbetreuung und Kommunikation), Professor Stefan Schönberg (DRG-Präsident), Andy Rogers (BIR-Präsident), Simon Thompson (CEO des BIR), Stefan Lohwasser (Geschäftsführer der DRG). Foto: BIR

\section{SO FUNKTIONIERT'S:}

Damit Sie die Inhalte des BIR nutzen könnten, müssen Sie sich einmalig kostenlos beim BIR registrieren. In der Registrierung geben Sie an, dass Sie DRG-Mitglied sind. Den Link zum jeweils aktuellen Beitrag erhalten Sie mit dem monatlichen DRG-Newsletter. 